\section{Staff and bed distribution in public sector mental health services in the Eastern Cape Province, South Africa}

\author{
K Sukeri, ${ }^{1} \mathrm{MB} \mathrm{ChB}, \mathrm{FCPsych}(\mathrm{SA}) ;$ O Alonso-Betancourt, ${ }^{1} \mathrm{MD}, \mathrm{PhD}, \mathrm{FCPsych}(\mathrm{SA})$; \\ R Emsley, ${ }^{2}$ MB ChB, DCH, MMed (Psych), FCPsych (SA), DMed, DSc \\ ${ }^{1}$ Department of Psychiatry, Faculty of Health Sciences, Walter Sisulu University, Mthatha, Eastern Cape, South Africa \\ ${ }^{2}$ Department of Psychiatry, Faculty of Medicine and Health Sciences, Stellenbosch University, Tygerberg, Cape Town, South Africa
}

Corresponding author: K Sukeri (ksukeri@telkomsa.net)

Background. The Eastern Cape Province of South Africa is a resource-limited province with a fragmented mental health service. Objective. To determine the current context of public sector mental health services in terms of staff and bed distribution, and how this corresponds to the population distribution in the province.

Method. In this descriptive cross-sectional study, an audit questionnaire was submitted to all public sector mental health facilities. Norms and indicators were calculated at provincial and district level. This article investigates staff and bed distribution only.

Results. Results demonstrated that within the province, only three of its seven districts have acute beds above the national baseline norm requirement of 13/100 000. The private mental health sector provides approximately double the number of medium- to long-stay beds available in the public sector. Only two regions have staff/population ratios above the baseline norm of 20/100 000. However, there are significant differences in this ratio among specific staff categories. There is an inequitable distribution of resources between the eastern and western regions of the province. When compared with the western regions, the eastern regions have poorer access to mental health facilities, human resources and non-governmental organisations.

Conclusion. Owing to the inequitable distribution of resources, the provincial authorities urgently need to develop an equitable model of service delivery. The province has to address the absence of a reliable mental health information system.

S Afr J Psychiatr 2014;20(4):160-165. DOI:10.7196/SAJP.570

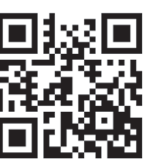

The Eastern Cape (EC) Province is located on the south eastern coast of South Africa (SA). The province has an approximate geographical area of $169580 \mathrm{~km}^{2}$, which represents $14 \%$ of the country's land area. ${ }^{[1]}$ It was formed by the amalgamation of the two former homelands, Transkei and Ciskei, with the border regions of East London and Port Elizabeth (both former Cape Provincial Administration). At the time of this study (2010), the province was divided into one metropolitan region and six districts with 38 municipalities. ${ }^{[2]}$

The population of the province at the time of this study was $6527747 .^{[1]}$ Black Africans made up the majority of the population (88\%), and females constituted $52 \%$ of the total population. The high proportion of women is a reflection of the migrant labour system prevalent in this province. A total of $33 \%$ of the population was $<15$ years old, and those $\geq 65$ years constituted $6 \%{ }^{[1]}$

A Statistics SA report, based on a household survey measuring income and expenditure, ranked the EC as the second poorest province in the country, with a poverty rate of $58 \% \cdot{ }^{[3]}$ As early as 2004 it was noted that seven of SA's ten poorest municipalities were located in the EC. The poorest municipality was Ntabankulu, with $85 \%$ of its population living below the poverty line. ${ }^{[4]}$ The upper limit of the poverty line was defined as R593/person/month.
In 2006 the Gini co-efficient (a measure of statistical dispersion intended to represent the income distribution of a population; in a situation of perfect equality, the co-efficient would equal zero) for the EC was 0.65 . The Western Cape had a co-efficient of 0.58 and the national figure for the same period was $0.64 .^{[5]}$ This demonstrates the vast inequality that exists interprovincially, a remnant of the policies of apartheid. As of the first quarter of 2009, the unemployment rate was $28 \%$, the highest in the country. ${ }^{[2]}$ At the time of the study, $57 \%$ of the population were dependent on social grants, ${ }^{[4]}$ and $\sim 73 \%$ of the province's children lived in households with an income of $\leq \mathrm{R} 800$ per month. ${ }^{[6]}$

Approximately $90 \%$ of the population was dependent on public sector health services. The HIV prevalence rate among antenatal attendees was $29.3 \%$ in $2011 .{ }^{[7]}$ The prevalence rate among 15 49-year-olds was recorded at $19 \%$ in $2012 .{ }^{[8]}$ The province has the third highest number of new infections in the country, with an estimated annual incidence rate of $1.5 \%{ }^{[9]}$

The policy of apartheid ensured that health services in SA were segregated by race, and mainly centralised in large urban areas. ${ }^{[10]}$ The homelands were underserviced. The result was an inequitable health system. This is apparent in the EC, where mental health services are disjointed and fragmented. The former homelands have poorer access 
to public sector mental health facilities and no private facilities. Private sector facilities are only available in the two former nodes of urban growth within apartheid SA, viz. Port Elizabeth and East London.

The province's public sector mental health facilities consist of four psychiatric hospitals (one of which is the only long-stay facility in the province; one other provides mediumto long-stay beds) and six mental health units (attached to tertiary hospitals). There are no public sector community residential psychiatric facilities, and the province has limited primary mental healthcare facilities.

There is no provincial policy on mental health. Mental health is incorporated into the general strategic plan for the entire province. In 2007, the provincial Department of Health (DoH), in filling in the World Health Organization (WHO)'s Assessment Instrument for Mental Systems (WHOAIMS) country report for SA, was unable to provide information in several domains. These included policy and plans, financing of mental health and human rights policies in domain one. In domain four there were errors in reporting of staff numbers. The provincial DoH was also unable to provide data on service utilisation, training of nurses at primary healthcare level and intersectoral collaboration. ${ }^{[11]}$ The key, therefore, would be to transform the current system to provide an equitable and efficient mental health system, planned according to well-informed and valid statistics.

The objective of this study was to investigate the current distribution of staff and beds in the public sector mental health service in the province.

\section{Methods}

In this descriptive cross-sectional study, a questionnaire adapted from the norms manual for severe psychiatric disorders ${ }^{[12]}$ and the WHO-AIMS ${ }^{[13]}$ were used to collect both quantitative and qualitative data. Ethics approval for the study was obtained from the Walter Sisulu University Health Research Ethics Committee.

Quantitative data collected included: categories of staff; number of staff in each category; whether staff were part- or fulltime; hours spent on clinical, academic and research output; admissions and discharges; and the number of acute and medium- to long-term beds. Acute beds were defined as
$<3$ months' stay, while medium- to long-term beds were $\geq 3$ months' stay. ${ }^{[12]}$

Qualitative data focused on communication with the Directorate of Specialised Services, which is the provincial authority responsible for the provision of mental health services, functioning of the Mental Health Review Boards, compliance of district hospitals in completion of the Mental Health Care Act forms and 72-hour assessments, access to psychotropic medication, outreach, and education (if provided) to primary healthcare staff.

The first step was to survey all mental health hospitals and units in the province. The hospitals were identified as per the district in which they are located (Table 1). The questionnaire was distributed to all psychiatric hospitals and units, to be completed by the resident psychiatrist/ hospital manager. To control for nonresponse bias, the data were cross-checked with assistant managers/nurse managers, and where discrepancies existed, the resident psychiatrist was contacted to clarify the data.

The next step was to determine the population size of each of the districts, as this would be the denominator in calculating staff and bed population ratios. The data were then entered into an Excel spreadsheet document, designed to calculate staff and bed population ratios per region. The province does not have a child and adolescent service; this is provided by general psychiatrists. Therefore, the entire population was used in all calculations.

The baseline workforce need was calculated utilising the formula set out by Scheffler et al. ${ }^{[14]}$ According to these authors, SA (a middle-income country) requires a mental health workforce of 26.7/100 000 .

Mental health services for the Alfred Nzo district are provided by staff in the OR

Table 1. Distribution of hospitals and mental health units in the Eastern Cape

\begin{tabular}{lll}
\hline District & Psychiatric hospital & Mental health unit \\
\hline Alfred Nzo & No & No \\
Amathole & Yes (Tower Hospital)* & 1 unit (East London) \\
Cacadu & Fort England & No \\
Chris Hani & Komani & No \\
Nelson Mandela Metropolitan & Elizabeth Donkin & 2 units (Dora Nginza, Uitenhage) \\
OR Tambo & No & 3 units (Mthatha, Libode, Flagstaff) \\
Ukhahlamba & No & No \\
${ }^{*}$ Tower Hospital is a long-stay facility. & &
\end{tabular}

Tambo district; therefore, in calculating staff/ population ratios, the populations of both districts were added. Similarly, the districts of Nelson Mandela Metropolitan and Cacadu provide services to Ukhahlamba; therefore, the population for the latter district was equally divided between the two.

Data analysis followed the WHO's recommendations ${ }^{[14,15]}$ in addition to the norms manual for severe psychiatric disorders. ${ }^{[12]}$ In this manual, pages 22 - 76 clearly outline the stepwise calculations. In the current article, the authors were interested only in bed distribution and human resources available. Staff/population ratios were calculated on 100\% full-time equivalents, which were defined as the number of staff who worked full-time, and included percentages of staff who spent only some of their time in mental health settings. ${ }^{[12]}$ The forensic facility at Fort England Hospital was not included in this article, as it is dealt with separately as a specialised service.

\section{Results}

The results section commences with psychiatric hospital and mental health unit location, followed by bed and staff distribution. A comparison with baseline norms is included in the tables.

Results for the public sector psychiatric bed distribution and bed/population ratio for each of the eight regions in the province are shown in Table 2. Staff distribution per district in the province and staff population ratios are represented in Tables 3 and 4, respectively.

Although staff/population (Table 4) ratios were calculated at $100 \%$ full-time equivalents, this is not a correct representation of the clinical time available for psychiatrists, registrars and medical officers, as they are involved in additional outreach, academic and 
research activities. In addition, psychiatrists employed at the principal psychiatrist level are required to perform administrative duties. Psychiatrists in the province utilise $60 \%$ of their time for clinical duties (which include inpatient and outpatient care), $30 \%$ for administrative duties and $10 \%$ for teaching of undergraduate and postgraduate students.

From Table 4 it is evident that only the districts of Cacadu and Chris Hani met the baseline norm of 24 for the total number of nurses. ${ }^{[12]}$ These districts also met the baseline norm of 20 for total clinical staff. The provincial total clinical staff ratio was 14.15/100 000, which does not meet either baseline (20.1) or target (35.9) norms. This represents a shortfall of 391 total clinical staff at baseline norm and 1419 at target norm.

The baseline workforce needed for the EC, according to Scheffler et al., ${ }^{[14]}$ translates to 645.84 nurses (54\%) working in mental health settings, 71.76 (6\%) psychiatrists and 490.36 (41\%) staff for psychosocial services (including psychologists, social workers and occupational therapists). The human resource calculation is for the provision of an adult mental health service for outpatients (acute and primary) and inpatients (acute and long-stay care). In complying with WHO recommendations, Scheffler et al. ${ }^{[14]}$ excluded registrars and medical officers, as they could inflate the supply.

The staff/bed ratio for the province was 0.77 , more than double the baseline norm of 0.36. A closer analysis of the staff/bed distribution is shown in Table 5 .

\section{Discussion}

There is an urgent need to reassess public sector mental health bed distribution within the province (Table 2). Only three districts have acute mental health beds above the baseline norm of $13,{ }^{[12]}$ viz. Cacadu, Nelson

Table 2. Public sector psychiatric beds per region, $n$ (bed/population ratios)

\begin{tabular}{lll}
\hline District & Acute beds & Medium- to long-stay beds \\
\hline Alfred Nzo & $0(0 / 100000)$ & $0(0 / 100000)$ \\
Amathole & $40(5.10 / 100000)$ & $0(0 / 100000)$ \\
Cacadu & $70(19.25 / 100000)$ & $10(2.75 / 100000)$ \\
Chris Hani & $180(22.54 / 100000)$ & $250(31.3 / 100000)$ \\
Nelson Mandela Metropolitan & $163(15.51 / 100000)$ & $0(0 / 100000)$ \\
OR Tambo & $93(4.99 / 100000)$ & $0(0 / 100000)$ \\
Ukhahlamba & $0(0 / 100000)$ & $0(0 / 100000)$ \\
Tower Hospital & 45 & $355(5.44 / 100000)$ \\
Total & $591(9.05 / 100000)$ & $615(9.42 / 100000)$
\end{tabular}

Mandela Metropolitan and Chris Hani. All these districts are in the western region of the province. The two districts with zero beds for mental health care are located in the eastern region. None of the regions met the target norm of $28 .{ }^{[12]}$ It must be noted that although the number of acute beds in the Amathole region had increased by 10 in 2012 (increasing to $5.71 / 100000$ ), the bed distribution in the region and the province remained below both baseline and target norms. The shortfall for acute beds in the province was 228 and 1206 beds at baseline and target norms, respectively. The shortfall was mainly in the border and eastern regions of Amathole and OR Tambo, respectively.

When comparing with national and international figures, a consensus definition has to be utilised in determining communitybased inpatient beds. The WHO defines these beds as units located within general hospitals outside of mental hospitals. ${ }^{[13]}$ Utilising this definition, the EC therefore has 2.7/100 000 community-based inpatient beds. This is almost double that of Uganda $(1.4 / 100000)^{[16]}$ and six times that of Nigeria $(0.45 / 100000){ }^{[17]}$ The national figure for SA is $2.8 / 100000 \cdot{ }^{[11]}$ Although the EC figure appears to indicate parity with the national figure and above-average provision when compared with other African countries, it must be noted that the distribution of beds is extremely unequal between the eastern and western regions of the province, as indicated in Table 5. In some units and hospitals,

Table 3. Staff distribution per district, $n$

\begin{tabular}{|c|c|c|c|c|c|c|c|c|c|}
\hline Staff & Alfred Nzo & Amathole & Cacadu & Chris Hani & NMM & ORT & UKH & Tower & Total \\
\hline Professional nurse & 0 & 31 & 103 & 85 & 17 & 45 & 0 & 34 & 315 \\
\hline Other nurse & 0 & 49 & 85 & 113 & 60 & 34 & 0 & 153 & 494 \\
\hline OT & 0 & 1 & 2 & 1 & 1 & 0 & 0 & 2 & 7 \\
\hline OTA & 0 & 1 & 3 & 2 & 3 & 1 & 0 & 0 & 10 \\
\hline SW & 0 & 3 & 3 & 5 & 3 & 4 & 0 & 3 & 21 \\
\hline CHW & 0 & 0 & 0 & 0 & 0 & 0 & 0 & 0 & 0 \\
\hline Psychologist & 0 & 1 & 18 & 3 & 4 & 1 & 0 & 1 & 28 \\
\hline Intern psychologist & 0 & 1 & 7 & 0 & 0 & 0 & 0 & 0 & 8 \\
\hline Psychiatrist & 0 & 2 & 3 & 1 & 5 & 3 & 0 & 0 & 14 \\
\hline Registrar & 0 & 0 & 2 & 0 & 1 & 5 & 0 & 0 & 8 \\
\hline Medical officer & 0 & 4 & 5 & 4 & 3 & 1 & 0 & 3 & 20 \\
\hline Total & 0 & 93 & 231 & 214 & 96 & 94 & 0 & 196 & 924 \\
\hline
\end{tabular}


physical beds may be present but are not utilised for various reasons, including the non-availability of mattresses and linen.

The public sector bed/population ratio is substantially lower than that in First-World countries such as the USA, where the recommended ratio is $50 / 100000 .{ }^{[18]}$ The province has a shortfall of 434 public sector medium- to long-term beds and 540 private sector beds. These figures are at the baseline norm (public sector 16 , private sector 19). ${ }^{[12]}$ Currently, Life Esidemeni, a public/private partnership, provides 805 private sector medium- to long-term stay beds and 94 acute beds. The medium- to long-term stay beds comprise 666 adult and 139 children and adolescent beds at two separate facilities. Acute beds are located in Port Elizabeth and East London only. When the children in these facilities become adults, they are transferred to the adult wards. ${ }^{[19]}$

The only non-governmental organisations that provide community residential care are located in Nelson Mandela Metropolitan. These organisations provide a total of 160 residential beds, ${ }^{[20,21]}$ including beds for adults with learning disabilities. This translates to $2.45 / 100000$ beds provincially, which compares poorly with the national figure of $3.6 / 100000 .^{[1]}$
In 2001, with the transfer of Umzimkulu Hospital to KwaZulu-Natal, the province effectively lost 440 medium- to long-term stay beds. ${ }^{[22]}$ This hospital was located in the eastern region and provided a much-needed service there. In addition to bed loss, there was also a movement of essential staff and services to KwaZulu-Natal, including psychological, occupational therapy, social and rehabilitative services. The combined loss of staff and beds resulted in an increased migration of mental healthcare patients to the western region, increasing the burden on services there. The provincial DoH did not plan for this loss of service in the eastern region, as there was no increase in services in the western region to meet the increased demand, and planned services for the eastern region were not completed until 2005, when the number of acute beds increased from 20 to 60 in Mthatha (personal communication with the Head of Department of Psychiatry, Walter Sisulu University; May 2010).

As none of the psychiatric hospitals are able to accommodate mentally ill children and adolescents, these patients are admitted either to paediatric or general medical wards. Children and adolescents requiring long-term care are transferred to the public/ private facility in Port Elizabeth.

Table 4. Staff/population ratios per district

\begin{tabular}{|c|c|c|c|c|c|c|c|}
\hline & $\begin{array}{l}\text { Baseline } \\
\text { norm }\end{array}$ & $\begin{array}{l}\text { AN } \\
\text { and ORT }\end{array}$ & Amathole & $\begin{array}{l}\text { Cacadu } \\
\text { and UKH }\end{array}$ & $\begin{array}{l}\text { Chris } \\
\text { Hani }\end{array}$ & $\begin{array}{l}\text { NMM } \\
\text { and UKH }\end{array}$ & Tower \\
\hline Total nurses & 24 & 3.37 & 4.80 & 36.32 & 24.79 & 6.39 & 2.86 \\
\hline OT & 0.4 & 0.00 & 0.06 & 0.39 & 0.13 & 0.08 & 0.03 \\
\hline OTA & 0.9 & 0.00 & 0.06 & 0.58 & 0.25 & 0.25 & 0.00 \\
\hline SW & 0.7 & 0.17 & 0.18 & 0.58 & 0.63 & 0.25 & 0.05 \\
\hline CHW & & 0.00 & 0.00 & 0.00 & 0.00 & 0.00 & 0.00 \\
\hline Psychologist & 0.3 & 0.04 & 0.06 & 3.48 & 0.38 & 0.33 & 0.02 \\
\hline Intern psychologist & & 0.00 & 0.06 & 1.35 & 0.00 & 0.00 & 0.00 \\
\hline Psychiatrist & 0.3 & 0.13 & 0.12 & 0.58 & 0.13 & 0.41 & 0.00 \\
\hline Registrar & 0.4 & 0.21 & 0.00 & 0.39 & 0.00 & 0.08 & 0.00 \\
\hline Medical officer & 0.4 & 0.04 & 0.24 & 0.97 & 0.50 & 0.25 & 0.05 \\
\hline Total & 20.1 & 3.96 & 5.64 & 44.64 & 26.81 & 8.04 & 3.01 \\
\hline
\end{tabular}

Table 5. Staff/bed ratios per district

\begin{tabular}{|c|c|c|c|c|c|c|c|c|c|c|}
\hline Staff & BN & Prov & AN & AT & C & $\mathrm{CH}$ & NMM & ORT & UKH & Tower \\
\hline Total nurses & 0.25 & 0.41 & 0.00 & 0.5 & 2.35 & 0.47 & 0.47 & 0.85 & 0.00 & 0.47 \\
\hline Psychiatry nurses & 0.12 & 0.26 & 0.00 & 0.8 & 1.29 & 0.2 & 0.04 & 0.48 & 0.00 & 0.09 \\
\hline OT & 0.01 & 0.01 & 0.00 & 0.05 & 0.01 & 0.00 & 0.01 & 0.00 & 0.00 & 0.01 \\
\hline OTA & 0.02 & 0.00 & 0.00 & 0.03 & 0.04 & 0.00 & 0.02 & 0.01 & 0.00 & 0.00 \\
\hline SW & 0.01 & 0.02 & 0.00 & 0.08 & 0.04 & 0.00 & 0.02 & 0.04 & 0.00 & 0.01 \\
\hline Psychologist & 0.01 & 0.02 & 0.00 & 0.03 & 0.23 & 0.01 & 0.02 & 0.01 & 0.00 & 0.00 \\
\hline Psychiatrist & 0.01 & 0.01 & 0.00 & 0.05 & 0.04 & 0.00 & 0.03 & 0.03 & 0.00 & 0.00 \\
\hline Registrar & 0.01 & 0.01 & 0.00 & 0.00 & 0.03 & 0.00 & 0.01 & 0.05 & 0.00 & 0.00 \\
\hline Medical officer & 0.01 & 0.02 & 0.00 & 0.1 & 0.06 & 0.01 & 0.02 & 0.03 & 0.00 & 0.01 \\
\hline
\end{tabular}


Staff/population and staff/bed ratios serve as important indicators of a region's ability to meet the mental health needs of its population. These ratios are valuable in planning and resource allocation in any health service. They have a direct impact on the level of care that can be provided within a health system. In psychiatric hospitals, this may translate to the ability to manage high-risk patients, such as suicidal and homicidal patients in both secure and non-secure wards.

From the results provided in Tables 4 and 5 , it can be seen that the provincial figures for clinical staff and staff/bed ratios are misleading. Both these tables clearly demonstrate a maldistribution of staff and shortages in specific staff categories. For example, Nelson Mandela Metropolitan meets the baseline norm for total number of nurses, but closer analysis (Table 5) demonstrates a shortage of nurses trained in psychiatry, a vital component of a multidisciplinary team. A similar result is demonstrated for Tower Hospital. Cacadu is the only district that meets the baseline norm for all categories of staff.

There is a critical shortage of nurses with advanced psychiatry training in the province. Enrolled nurses provide a supportive role to professional nurses. Combining these staff categories inflates the total number of nurses and therefore results in an erroneous ratio.

It must be noted that staff/bed ratios have improved since an earlier report by Lund et al. ${ }^{[23]}$ in 2001, who reported a total staff/bed ratio of 0.3 ; in 2010 this figure increased to 0.75 . There have been significant increases in specific staff categories, viz. psychiatrists, registrars, social workers and psychologists; however, there has been a significant drop in the number of beds, from 2330 to 1231 , which has affected the staff/bed calculation. If the status quo had remained, the staff/bed ratio would have increased to 0.39 , which is not a significant increase. In 2001, the psychiatrist/population ratio for the EC was 0.2, based on the total population derived from the 1996 census. ${ }^{[24]}$ In 2010 , a research paper reported a ratio of $0.1 / 100000$, based on the calendar year 2005. ${ }^{[25]}$ According to the authors' calculation, this ratio had increased to 0.25 for the year 2010. The lack of accurate data is a reflection of the need for a reliable mental health information system in the province. Lund et al. ${ }^{[25]}$ reported that the national ratio for psychiatrists was $0.28 / 100000$ in 2010. In comparison, the province of KwaZulu-Natal had a ratio of 0.38 for the same period. ${ }^{[26]}$ In 2012, the ratio for the EC remained 0.25. This demonstrates the interprovincial differences in resources available in the public mental health sector in SA.

According to WHO recommendations, the EC has a shortfall of 58 psychiatrists and an oversupply of 163 nurses. It must be noted, however, that nurses trained in psychiatry provide an essential service where there are no specialists or trained medical officers in mental health. The WHO study did not distinguish between nurses trained in psychiatry and enrolled nurses, as their roles differ significantly within the health system. ${ }^{[15]}$

Bruckner et al. ${ }^{[15]}$ reported that in low- to middle-income settings, 1.2 psychiatrists per 100000 population are required to manage medium-stay residential centres, acute inpatient care and an outpatient and primary care centre. This translates to seven psychiatrists managing these clinical requirements in the EC. This study did not consider rural v. urban settings, distances to travel and that psychiatrists in low-income settings perform administrative, research and teaching duties in addition to clinical work. Adjustment for these factors would increase the number of these skilled professionals required in the province. There are no medium-stay facilities in the EC that are attended to by psychiatrists; all of these facilities are run by non-governmental organisations. This doubles the need for psychiatrists in clinical settings only, as outlined by Bruckner et al. ${ }^{[15]}$ In a resource-limited setting such as the EC, this means an overextension of the current workforce, resulting in a very limited coverage of mental health requirements.

The private sector in the province provides services for $10 \%$ of the population, therefore staff/population and bed/population ratios must be adjusted to reflect this. When this adjustment is effected, the staff/population ratio is not affected; however, the bed ratios for acute beds (10.57/100 000) and medium- to long-term beds (10.38/100 000) improve. Comparatively, the ratios for the private sector in the EC are $1.7 / 100000$ (staff/population) and 14.4/100 000 (bed/population). These ratios for the private sector will have implications in the design of the proposed National Health Insurance, where private sector resources will align with public sector facilities.

In previous research, ${ }^{[23]}$ it has been reported that in 2001, the province had a total workforce in mental health services of 859 , which at that time resulted in a staff/population ratio of 14.42/100 000. This figure also included 57 community health workers (CHWs) in the province. In 2010, 9 years later, there were no CHWs in the province. To date (2012), there are no CHWs in the public sector mental health service. This again is a reflection of a poor mental health information system or information bias. CHWs would form an integral component in a psychosocial rehabilitation programme. The contribution by this category of staff would decrease the need for psychiatric nurses conducting both tertiary and community services.

\section{Conclusion}

It is evident that there is an inequitable distribution of resources between the eastern and western regions of the EC. This may be a remnant of SA's apartheid past; however, this critical issue can be addressed with informed planning and policy development. Central to this would be the development of an efficient mental health information system in this province.

A well-planned mental health information system, comprising trained personnel in data collection methods, would collate both quantitative and qualitative data and provide health authorities with the information required to determine local needs, resource allocation and target setting. ${ }^{[27]}$ Resource allocation can be addressed by improving funding for staff and beds in the eastern region. In addition, training of primary healthcare staff could alleviate the burden on secondary and tertiary services. The western region could develop a service more in line with international requirements, including the development of public sector community health services.

The province has to reach consensus in licensing private institutions to implement the Mental Health Care Act No. 17 of 2002; $;^{[28]}$ this will improve compliance with current regulations and improve intersectoral collaboration.

The presence of an academic Department of Psychiatry would provide the province with expertise in research and training of the mental health workforce. This will assist in strengthening primary and district services and decrease the burden on tertiary services. 
Imperative is the development of a mental health policy. This policy will require the involvement of stakeholders from all sectors of government and non-governmental organisations.

The recommendations above are in line with the recently published National Mental Health Policy Framework and Strategic Plan 2013 - 2020. ${ }^{[29]}$ This national plan sets out guidelines for provincial departments to develop policy and plans within a specified timeframe. Utilising this guideline, and with careful planning, the EC is poised to develop a comprehensive strategy to address its shortcomings with staff and bed distribution, adapted to the needs of and available resources within the province.

Acknowledgement. This research was sponsored by a Discovery Academic Fellowship Grant.

\section{References}

1. Statistics South Africa. Community Survey 2007. http://www.statssa.gov.za (accessed November 2010).

2. Makiwane MO, Chimere-Dan ODD. The People Matter. The State of the Population of the Eastern Cape, 2010. Human Sciences Research Council. http://www.hsrc.ac.za/uploads/ pageContent/558/ECapefullreport.pdf (accessed December 2010)

3. Statistics South Africa. Poverty Profile of South Africa. Application of the Poverty Lines on the Living Conditions Survey 2008/9. http://www.statssa.gov.za (accessed December 2010).

4. Schwabe C. Fact Sheet: Poverty in South Africa. Human Sciences Research Council, July 2004 http://www.sarpn.org/documents/d000090 (accessed November 2010).

5. Armstrong P, Lekezwa B, Siebrits K. Poverty in South Africa: A Profile Based on Recent Household Surveys. Stellenbosch Economic Working Papers 04/08: A working paper of the Department of Economics and the Bureau for Economic Research at the University of Stellenbosch. http://www. ekon.sun.ac.za/wpapers/2008/wp042008/wp-04-2008.pdf (accessed May 2010).

6. Leatt A. Income Poverty of South Africa. South African Child Gauge, 2006:24-30. http://www ci.org.za (accessed May 2010).

7. South African Department of Health. The National Antenatal Sentinel HIV and Syphilis Prevalence Survey, South Africa, 2011. Pretoria: National Department of Health, 2011.

8. Van der Linde I. Plenary Session 3, 20 June 2013: HIV/AIDS in South Africa: At Last the Glas is Half Full. Human Sciences Research Council. http://www.hsrc.ac.za/en/media-briefs/hivaids-stis-and-tb/plenary-session-3-20-june-2013-hiv-aids-in-south-africa-at-last-the-glassis-half-full (accessed March 2014).

9. Eastern Cape Department of Health. Provincial Strategic Plan for HIV/AIDS, STIs and TB 2012-2016. Bhisho: Eastern Cape Department of Health, 2012.
10. African National Congress. Access to Healthcare in South Africa: A National Health Plan for South Africa. 30 May 1994. http://www.anc.org.za/show.php?id=257 (accessed May 2010).

11. World Health Organization. WHO-AIMS Country Report for South Africa, 2007. Geneva: World Health Organization, 2007

12. Flisher AJ, Lund C, Muller L, et al. Norms and Standards for Severe Psychiatric Conditions: A Report Submitted to the Department of Health, Tender No. GES 105/96-97. Pretoria: National Department of Health, 2003.

13. World Health Organization. WHO Assessment Instrument for Mental Health Systems Version 2.2. Geneva: World Health Organization, 2005

14. Scheffler RM, Bruckner TA, Fulton BD, et al. Human Resources for Mental Health: Workforce Shortages in Low and Middle Income Counties. Geneva: World Health Organization, 2011.

15. Bruckner TA, Scheffler RM, Shen G, et al. The mental health workforce gap in low and middle income countries: A needs based approach. Bull World Health Organ 2011;89:184-194. [http:// dx.doi.org/10.2471/BLT.10.082784]

16. Kigozi F, Ssebunnya J, Kizza D, Cooper S, Ndyanabangi S. An overview of Uganda's mental health care system: Results from an assessment using the World Health Organization's assessment for mental health systems (WHO-AIMS). Int J Ment Health Syst 2010;4(1):1-9. [http://dx.doi.org/10.1186/1752-4458-4-1]

17. World Health Organization. WHO-AIMS Country Report for Nigeria, 2006. Geneva: World Health Organization, 2006.

18. Torrey EF, Entsminger K, Geller J, Stanley J, Jaffe DJ. The Shortage of Public Hospital Beds for Mentally Ill Persons. A Report of the Treatment Advocacy Centre. http://www treatmentadvocacycentre.org (accessed February 2011).

19. Life Health Care. Mental healthcare facilities and services. http://www.lifehealthcare.co.za/ Hospital/Mental_Health_Services.aspx (accessed May 2010).

20. Care Haven Psychiatric, Residential and Care Centre, Port Elizabeth, South Africa. http:// carehavenpe.co.za (accessed May 2010).

21. Lake Farm Centre. A loving community for intellectually challenged adults. http://www. lakefarm.co.za (accessed May 2010).

22. KwaZulu-Natal Department of Health. Umzimkhulu Hospital. http://www.kznhealth.gov.za/ umzimkhuluhospital.htm (accessed May 2010)

23. Lund C, Flisher AJ. South African mental health process indicators. J Ment Health Policy Econ 2001;4(1):9-16

24. Lund C, Flisher AJ. Staff/population ratios in South African public sector mental health services. S Afr Med J 2002;92(2):161-164.

25. Lund C, Kleintjies S, Kakuma R, Flisher AJ. Public sector mental health systems in South Africa: Inter-provincial comparisons and policy implications. Soc Psychiatry Psychiatr Epidemiol 2010;45(3):393-404. [http://dx.doi.org/10.1007/s00127-009-0078-5]

26. Burns JK. Mental health service funding and development in KwaZulu-Natal: A tale of inequity and neglect. S Afr Med J 2010;100(10):662-666.

27. Wing J, Brewin CR, Thornicroft G. Defining mental health needs. In: Thornicroft G, ed. Measuring Mental Health Needs. London: Gaskell, 2001:1-21

28. South African Government. Mental Health Care Act No. 17 of 2002. Pretoria: Government Printer, 2004.

29. South African Department of Health. National Mental Health Policy Framework and Strategic Plan 2013-2020. Pretoria: Government Printer, 2012 